\title{
Image Restoration by DOG Multi-Scale Analysis
}

\author{
K. Honda*, K. Kawauchi*, T. Nokuo*, N. Kikuchi** and N. Erdman** \\ *JEOL Ltd., Tokyo196-8558, Japan \\ **JEOL USA, Inc., MA 01961
}

This paper describes a newly developed image restoration method, which uses a multi-scale analysis of an image with wavelet-like sub-band decomposition. In conventional wavelet methods, the decomposition has been done by the quadratic dilation of wavelet frame size, which is based on the pyramidal algorithm [1]. On the contrary, we separate multi-scale operators and a signal completely in the decomposition procedure and generate optimum 2D filters for images with a wide range of frequencies, which allows a simple structure and faster calculation. The application of this method to SEM images is depicted.

Image $\mathrm{J}$ can be divided into a blurred image and its differentiation shown in eq. (1).

$$
J=J_{0}=J_{n}+\left(G_{0}-G_{n}\right) \otimes J
$$

$G_{k}$ means a Gaussian distribution with a standard deviation $\sigma=\sigma_{k}$ and $\mathrm{k}$ is a suffix on the condition: $\sigma_{n}>\cdots>\sigma_{k}>\cdots>\sigma_{1}:(\mathrm{k}=1, \ldots, \mathrm{n}) . G_{0}$ means the Dirac delta function, then $J=G_{0} \otimes J\left(=J_{0}\right)$ and $\otimes$ means the convolution. We expand the second term into smaller differentiations and define each of them as an operator $M_{k}$ of 'k-th DOG kernel' shown in eq. (2). Conventionally $M_{k}$ is called as DOG (Differentiation of Gaussian) function [2], but we use it as an elemental kernel of frequency expansion shown in Fig.1.

$$
J=J_{n}+\left\{\left(G_{0}-G_{1}\right)+\left(G_{1}-G_{2}\right)+\cdots+\left(G_{n-1}-G_{n}\right)\right\} \otimes J=J_{n}+\left[\sum_{k=1}^{n} M_{k}\right] \otimes J .
$$

Our purpose is to transform the input image $J$ to an improved image $J^{*}$, that is, sharpened and edge enhanced. This can be done by setting coefficients $\beta_{k}$ of the k-th DOG kernel and $\alpha$ of the n-th blurred image $J_{n}$. Fitting these coefficients to heuristic evaluation functions is done statistically by maximizing the SNR (Signal to Noise Ratio) of the processed image with holding a natural image quality. By the definition of $M^{*} \equiv \alpha G_{n}+\sum \beta_{k} M_{k}, J^{*}$ can be re-written in eq. (3). It can be easily determined that $M^{*}$ is ank ${ }^{*}$ ptimum $2 \mathrm{D}$ filter and allows a faster calculation of the image processing rather than conventional ones.

$$
J^{*}=\alpha J_{n}+\left[\sum_{k=1}^{n} \beta_{k} M_{k}\right] \otimes J=M^{*} \otimes J .
$$

System configuration of our system is shown in Fig.2. In the block 'Dynamic Analysis', the optimum 2D filters and the power spectrums are calculated in pairs against for images with a wide range of spatial frequencies and are stored in (A) and (B) respectively. Ordinary, when an image $J$ is input to the block 'Static Analysis', pattern matching is done between the power spectrum of the image $J$ and the stored ones, 
which means the selection of an optimum 2D filter, and then the image $J^{*}$ is restored by a convolution of the selected optimum 2 D filter and the image $J$.

It is also possible to do multi-scale analysis. Several examples of SEM images (different values of $k$ ) are shown in Fig. 3 and the restored one is shown in Fig. 4, which is dramatically improved in edge enhancement. For SEM images of gold particle on carbon, the improved ratio of resolution, i.e. reso. $(J) / \operatorname{reso} .\left(J^{*}\right)$, is $1.3 \sim 3.5$ and 2.4 in average (resolution was measured by the FT method [3]). Calculation time is about 0.7 sec for 1280 x 1024 pixels of an image with $2.93 \mathrm{GHz}$ Intel(R) Core(TM)2 Duo CPU.

\section{References:}

[1] S. G. Mallat, IEEE. Trans. Pattern Analysis. Vol. 11. (7) (1989) 674.

[2] D. Marr et al., Proc. R. Soc. Lond. B 207 (1980) 187.

[3] To be published as ISO/TS 24597.
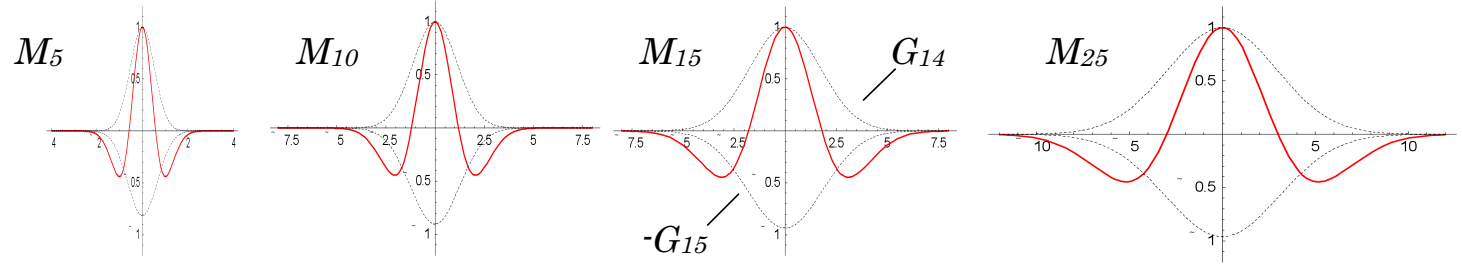

Fig. 1 Several examples of DOG kernels (normalized); $M_{k}(\mathrm{k}=5,10,15,25)$.
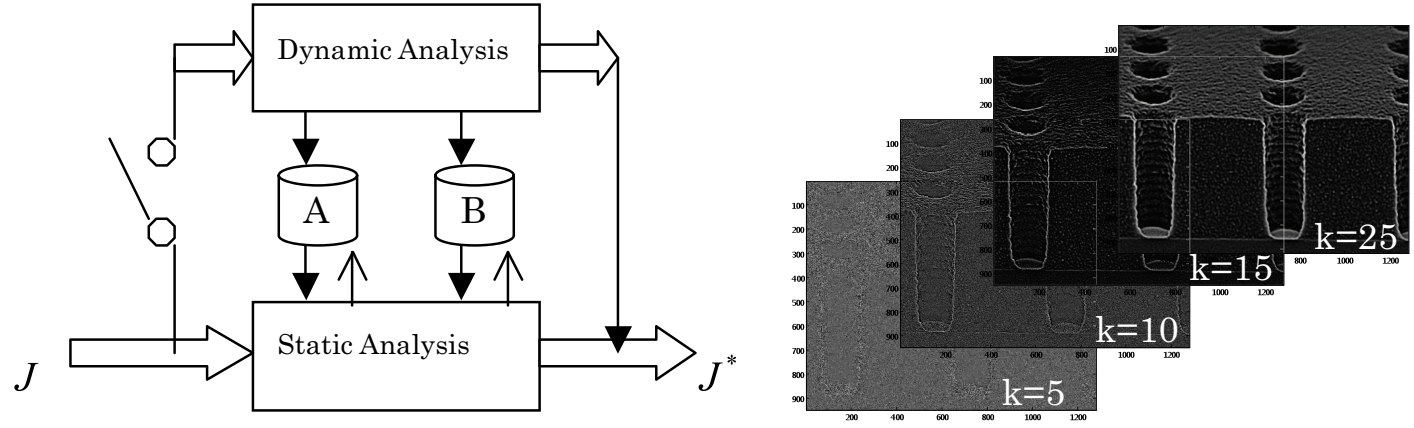

Fig. 2 System configuration.

Fig. 3 Several examples of multi-scale images.
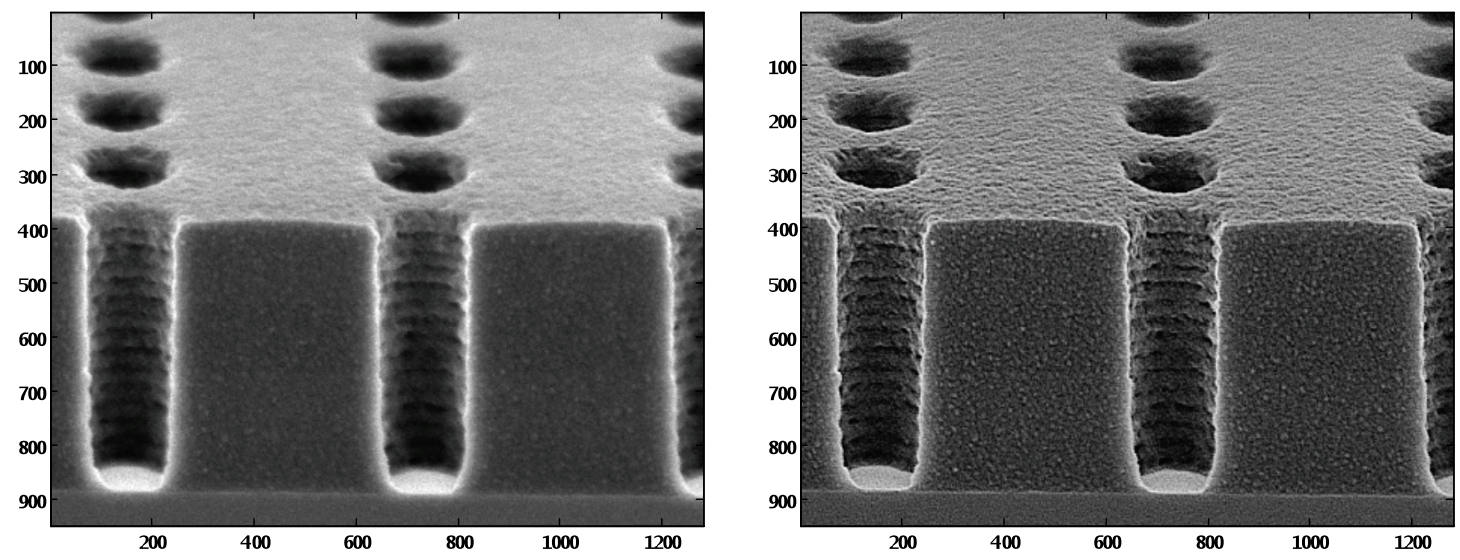

Fig. 4 Original image (left) and processed image (right): cross-section of contact holes. 